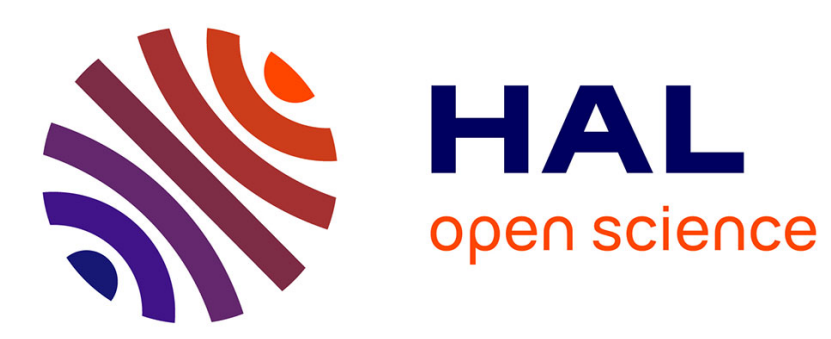

\title{
Digital Predistortion for CO-OFDM Systems Using Generalized Memory Polynomials
}

Jacqueline E Sime, Pascal Morel, Mihai Telescu, Noël Tanguy, Stéphane Azou

\section{To cite this version:}

Jacqueline E Sime, Pascal Morel, Mihai Telescu, Noël Tanguy, Stéphane Azou. Digital Predistortion for CO-OFDM Systems Using Generalized Memory Polynomials. 2020 IEEE Eighth International Conference on Communications and Electronics (ICCE), Jan 2021, Phu Quoc Island (virtual), Vietnam. pp.7-11, 10.1109/ICCE48956.2021.9352081 . hal-03148309

\section{HAL Id: hal-03148309 \\ https://hal.science/hal-03148309}

Submitted on 22 Feb 2021

HAL is a multi-disciplinary open access archive for the deposit and dissemination of scientific research documents, whether they are published or not. The documents may come from teaching and research institutions in France or abroad, or from public or private research centers.
L'archive ouverte pluridisciplinaire HAL, est destinée au dépôt et à la diffusion de documents scientifiques de niveau recherche, publiés ou non, émanant des établissements d'enseignement et de recherche français ou étrangers, des laboratoires publics ou privés. 


\section{Digital Predistortion for CO-OFDM Systems Using Generalized Memory Polynomials}

\author{
Jacqueline E. Sime \\ École Nationale d'Ingénieurs de Brest \\ Lab-STICC, CNRS, UMR 6285 \\ CS $73862-29238$, Brest Cedex 3, \\ France \\ jacqueline.sime@enib.fr \\ Noël Tanguy \\ Univ Brest \\ Lab-STICC, CNRS, UMR 6285 \\ F-29200 Brest, France \\ noel.tanguy@univ-brest.fr
}

\author{
Pascal Morel \\ École Nationale d'Ingénieurs de Brest \\ Lab-STICC, CNRS, UMR 6285 \\ CS $73862-29238$, Brest Cedex 3, \\ France \\ pascal.morel@enib.fr \\ Stéphane Azou \\ École Nationale d'Ingénieurs de Brest \\ Lab-STICC, CNRS, UMR 6285 \\ CS 73862 - 29238, Brest Cedex 3, \\ France \\ stephane.azou@enib.fr
}

\author{
Mihai Telescu \\ Univ Brest \\ Lab-STICC, CNRS, UMR 6285 \\ F-29200 Brest, France \\ mihai.telescu@univ-brest.fr
}

\begin{abstract}
Generalized memory polynomials have recently been proposed as an efficient means of linearization in wireless radio systems. Their unique structure provides an excellent compromise between complexity and accuracy. In the present paper, we show that the approach can successfully be transposed to optical systems. The investigated scenario is that of a CO-OFDM solution for metropolitan/access networks.
\end{abstract}

Keywords- digital predistortion, CO-OFDM, Semiconductor optical Amplifier, generalized memory polynomial, linearization

\section{INTRODUCTION}

The information age was brought about by significant advances in various fields of science. Photonics quickly established itself as the solution for transoceanic and transcontinental long-haul while radio turned out to be the driving force behind metropolitan and access networks. It was in radio communications, particularly with the emergence of wireless solutions, that the need for digital predistortion (DPD) appeared. Essentially, the competition for bandwidth coupled with budget constraints pushed the engineering community to look for solutions to reduce the impact of nonlinear effects intrinsic to power amplifiers on modulated signals. Indeed, power amplifiers, particularly in affordable, massively available solutions are major sources of nonlinearities. Digital predistortion aims to compensate these nonlinearities by adequately altering the modulated signals and various solutions have been proposed over the years (see [1] and the references therein).

More recently however the historical separation between long-haul communications relying on fiber optics, on one hand, and metropolitan and access networks relying on copper and wireless radio, on the other hand, has become less distinct. Optical access networks are now a reality for millions of users enabling throughputs at speeds never imagined before and an ever increasing variety of services. Thus a lot of the issues that had been encountered and solved in radio networks in the early 2000s start to emerge in photonics. For example the use of affordable amplification in access and metropolitan networks is becoming a hot topic in both academic and industry-related circles. Furthermore, recent literature shows increased interest in advanced optical modulation algorithms with Coherent Optical Orthogonal Frequency Division Multiplexing (CO-OFDM) being one possible solution; Digital predistortion has thus come to the attention of the photonics community [2]. With
Semiconductor Optical Amplifiers (SOAs) being a candidate for use in access networks [3], DPD is becoming a must particularly in a scenario using non-constant envelope modulation such as OFDM.

In the present paper the authors investigate some recent predistortion algorithms and test them in a scenario implementing CO-OFDM and using an SOA as a booster amplifier.

\section{Digital PREDistortion FOR OPTICAL MEtRopolitan ACCESS NETWORKS}

\section{A. The scenario of a CO-OFDM system using SOAs}

The architecture that will be explored in the context of this paper is depicted in Fig. 1. A data stream undergoes 4QAM modulation with each QAM symbol assigned to one of the 128 subcarriers belonging to one of 32 OFDM frames with a $5 \mathrm{GHz}$ bandwidth. An oversampling factor of 4 is used. The scenario includes a cyclic prefix equivalent to $\frac{1}{8}$ of each OFDM frame duration. Once the signal becomes analog after digital-to-analog conversion, it undergoes electro-optic conversion by means of a laser diode and an IQ modulator comprised of Mach-Zehnder modulators (MZMs) operating at null point with driving peak-to-peak voltages of $1.25 \times V_{\pi}$. The SOA, supplied by a $150 \mathrm{~mA}$ bias current $\left(I_{\text {bias }}\right)$, boosts

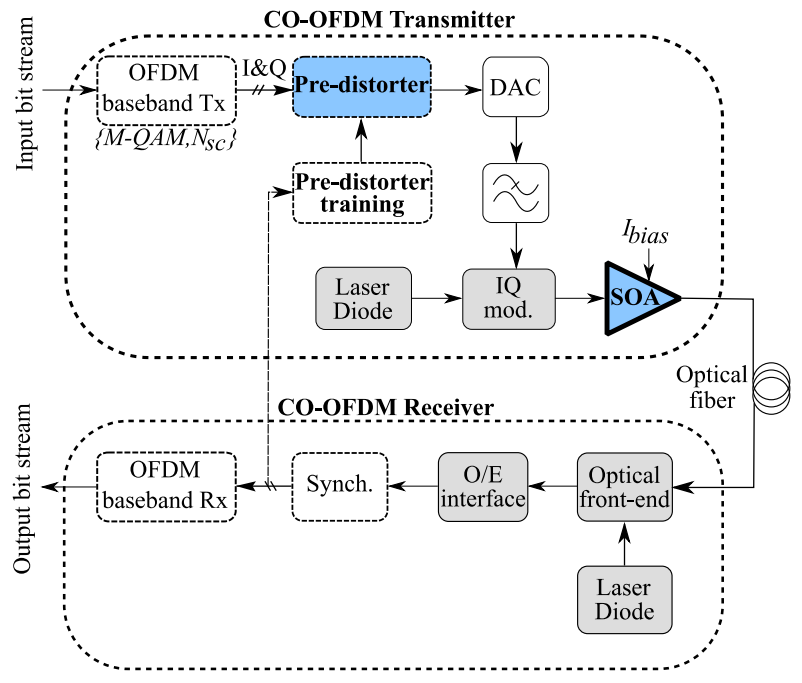

Fig. 1 CO-OFDM transmission system with a SOA and DPD 
the optical signal before it enters the fiber channel. By using a reference laser, photodetectors and trans-impedance amplifiers, the signal is able to get back into the electrical domain where after synchronization, the signal can be demodulated and the original data streams detected. The present paper focuses on nonlinearities mainly related to the SOA but also the IQ modulator. These will need to be compensated by the DPD while the other elements of the previously described system will be considered ideal at this stage.

However, it is important to gauge the fundamental differences that come about when switching from radiofrequencies to photonics and anticipate their influence. Several points bring interest to testing DPDs in photonics by spotting some uncommon grounds in RF and photonics. For instance, RF suffers from electromagnetic interference and propagation delay whereas photonics has to deal with chromatic dispersion and polarization mode dispersion. Furthermore, the addition of laser diodes and electro-optic and opto-electric conversion introduces some complications and limits the implementation of an adaptive algorithm unlike in RF. This means the DPD needs to be well optimized and robust from inception knowing that with the higher data rates associated with optics, the algorithms used to construct the DPDs should not be overly complex.

\section{B. Common predistortion solutions}

Predistortion relies on a relatively basic principle. One may see the channel as a nonlinear function altering the modulated signal in an undesired fashion. It appears quite natural to try to implement the inverse nonlinear function and add it into the system so that the effects of the two blocks simply cancel each other out. While the concept is simple, putting it into practice can prove quite complex.

In terms of identification two grand strategies are possible: direct learning and indirect learning. Direct learning implies building a mathematical model for the channel and analytically computing its inverse function. The approach is generally cumbersome and is not relevant to the scope of this paper. Indirect learning (shown in Fig. 2) bypasses the analytical inversion requirement by essentially recasting the problem as classical optimization. One starts with a well-chosen nonlinear parametric structure and subsequently computes its coefficients by minimizing an error criterion. Obviously the choice of the predistorter structure is crucial and non-trivial. However, a lot of relevant knowledge is already available in fields such as control theory or behavioral modeling where nonlinear system identification is a well explored topic.

Volterra Series is one of the oldest known techniques to describe nonlinear systems and is essentially a generalization of the impulse response function. Its mathematical expression is given by (1):

$$
y_{V}(n)=\sum_{k=0}^{K-1} \sum_{l_{1}=0}^{L-1} \ldots \sum_{l_{k}=0}^{L-1} a_{k, l_{1}, \cdots, l_{k}} \prod_{j=1}^{k} x\left(n-l_{j}\right)
$$

where $a_{k, l_{1}, \cdots, l_{k}}$ are the multidimentional Volterra kernels.

Traditionally, Volterra models are known to suffer from "the curse of dimensionality", their intrinsic complexity limiting their use to weakly nonlinear systems. Recently, progress in tensor decomposition has led researchers to

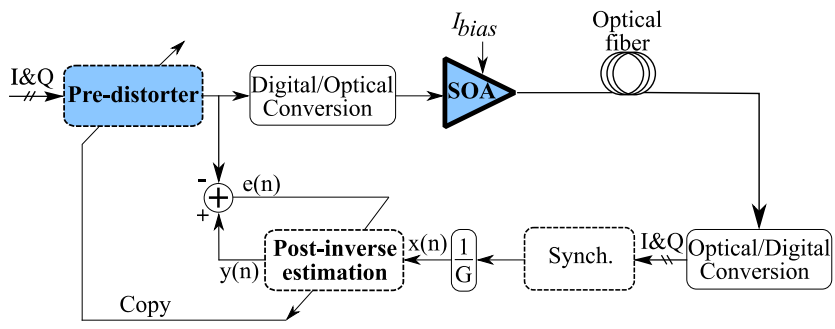

Fig. 2. Indirect learning approach

investigate the potential use of full-Volterra models in the context of DPD [4] but generally, simplified Volterrainspired structures are more common. Memory polynomials (MP) are typical examples of such structures which inputoutput relation is shown in (2).

$$
y_{M P}(n)=\sum_{k=0}^{K-1} \sum_{l=0}^{L-1} a_{k l} x(n-l)|x(n-l)|^{k}
$$

$K$ and $L$ are respectively the model's nonlinearity order and the memory depth. One notices the significant simplification achieved with respect to Volterra but practice shows there is a price to pay in terms of performance [4].

Further simplification can be achieved by the so-called envelope memory polynomials (EMP). EMP can be defined as shown in (3).

$$
y_{E M P}(n)=x(n)\left(c_{0}+\sum_{k=1}^{K-1} \sum_{l=0}^{L-1} a_{k l}|x(n-l)|^{k}\right)
$$

One may note that the main difference between MP and EMP is that in the case of the latter the nonlinearity of the system is related solely to the input's magnitude. Previous investigation shows that EMP is a better candidate for DPD [5] and the physics of the amplifier under test can explain these results.

From a practical point of view (2) and (3) can be rewritten in vector form as:

$$
y_{\text {model }}(n)=\boldsymbol{\varphi}_{\text {model }}(n) \boldsymbol{A}
$$

$\boldsymbol{A}$ contains the model coefficients and $\boldsymbol{\varphi}_{\text {model }}$ is a matrix constructed according to the input signal and the model. The coefficients can then be estimated by applying a least squares approach to (4).

In this paper, the MP and EMP will be used for the comparative analysis but there are many other DPD models that have been explored in recent research. For instance, Hammerstein and Wiener structures are also well known in nonlinear system identification as are their augmented versions such as FLUT [6]. For a more exhaustive review of DPD solutions one should refer to [1].

\section{Generalized Memory Polynomials}

Generalized memory polynomials (GMP) are a recent and elegant solution that includes MPs and EMPs while providing an extra degree of freedom. It should be noted that the generalization implied here is by no means a return to the Volterra series with its kernels that increase in dimension as the order of nonlinearity increases. This structure rather aims to take into account the signal's envelope, a lagging envelope and a leading envelope [7]. 


\section{A. Mathematical concept}

The equation describing the GMP model is as follows:

$$
\begin{aligned}
& y_{G M P}(n)=\sum_{k=0}^{K_{a}-1} \sum_{l=0}^{L_{a}-1} a_{k l} x(n-l)|x(n-l)|^{k} \\
& +\sum_{\substack{k=1 \\
K_{c}}}^{K_{b}} \sum_{\substack{l=0 \\
L_{c}-1}}^{L_{b^{\prime}}-1} \sum_{\substack{m=1 \\
M_{c}}}^{M_{b}} b_{k l m} x(n-l)|x(n-l-m)|^{k} \\
& +\sum_{k=1}^{K_{c}} \sum_{l=0}^{L_{c}-1} \sum_{m=1}^{M_{c}} c_{k l m} x(n-l)|x(n-l+m)|^{k}
\end{aligned}
$$

where $K_{a}, K_{b}$ and $K_{c}$ are the nonlinearity orders, and $L_{a}, L_{b}$ and $L_{c}$, and $M_{b}$ and $M_{c}$ are the memory depths. The coefficients $a_{k l}, b_{k l m}$, and $c_{k l m}$ are estimated in traditional manner using least squares.

\section{B. Parameter identification using the Hill-Climbing algorithm}

In the case of MP and EMP one needs to choose the order of nonlinearity and the memory depth, i.e. two parameter values. The choice is not trivial and one could even imagine testing 64 combinations of values assuming both the nonlinearity order and the memory depth are limited to a maximum value of 8 . While it does not seem completely impractical to do such comprehensive analysis for MP and EMP in the case of GMP the size of the problem becomes daunting; venturing into a grid search with $16,777,216$ combinations should obviously be ruled out for any practical purpose. One simple solution to this problem is given by the so-called Hill-Climbing algorithm which presents an automated means of selecting the structural GMP parameters [8]. The general stages of the HillClimbing algorithm can be described as listed below:

1. Create an 8-tuple initialized as a linear function with no memory $\left(L_{a}=K_{a}=L_{b}=M_{b}=L_{c}=M_{c}=1\right.$, $\left.K_{b}=K_{c}=0\right)$. This is subsequently referred to as "current point".

2. Search the neighborhood of current point. Here, the neighborhood is defined as the $(-1,0,1)$ range for each of the parameters.

3. Compute predistorders using (4) for entire neighborhood.

4. Compute the normalized mean square error (NMSE) for entire neighborhood.

5. Evaluate performance according to a criterion defined by $J=\lambda \times(\mathrm{NMSE}+20)+N_{\text {coeff }}$, with $N_{\text {coeff }}$ being the number of coefficients. (The criterion basically seeks a tradeoff between error level and number of coefficients.)

6. Rank all points in the neighborhood according to $J$. If current point has best $J$ stop; else point with best $J$ becomes "current point" and repeat 2-6.

In this paper, a value of $\lambda=4$ is chosen in order to put a higher weight on the number of coefficients. Note that there has also been research on automatically finding a desirable value for this constant [9].

\section{Simulation AND RESUlts}

Using the scenario described in section II with a commercial $750 \mu \mathrm{m}$ INPHENIX-IPSAD1501 SOA we perform a number of simulations comparing the performance of various DPD solutions. Note that the model used to simulate the system under test has been validated by measurements [10], [11].

\section{A. Efficiency analysis}

The objective of the first test is to compare a CO-OFDM scenario with no DPD with three scenarios featuring DPD (MP, EMP and GMP). Error vector magnitude (EVM) is computed for various values of the input power and the results are plotted in Fig. 3a. In 4-QAM OFDM on optical fiber access networks EVM values in the $30 \%$ range at the output of the booster are considered acceptable (this differs significantly from target levels in RF) [12]. Network reach is thus directly related to the highest input power that still guarantees the $30 \%$ EVM boundary. For each scenario, ten simulations featuring different OFDM sequences with 32 frames/sequence are carried out. The different plots in Fig. 3a show average EVM (dark thin lines) and standard deviation (shading). The predistorter coefficients are recomputed for each input power level. One notices that while MP does provide some improvement, EMP and GMP predistorters perform much better with GMP leading particularly at lower power levels. The analysis is repeated using normalized mean square error as a criterion (see Fig. $3 \mathrm{~b}$ ); the ranking of the various DPD variants is confirmed. It is interesting to observe that the standard deviation is reduced when DPD is included in the system (note thinner shading for blue, red and green plots). This entails that the system is more robust with respect to the characteristics of the input signal.
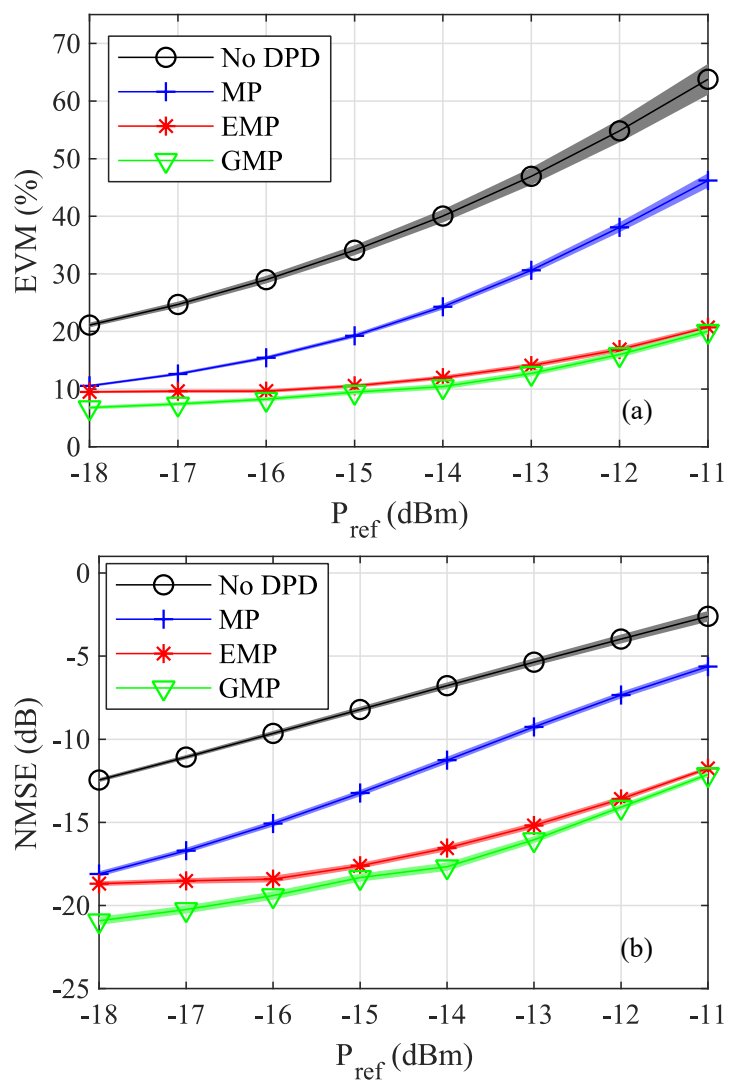

Fig. 3 Error Vector Magnitude (3.a) and Normalized Mean Square Error (3.b) at different input identification power 

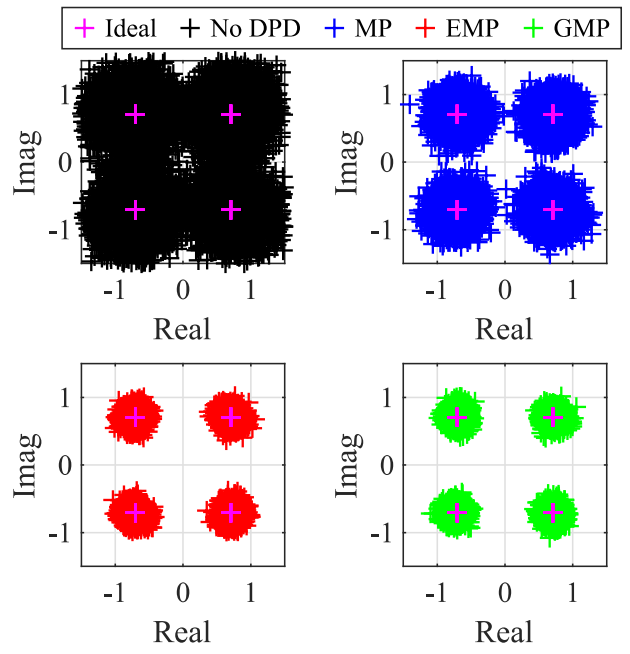

Fig. 4. Constellation at $P_{\text {ref }}=-14 \mathrm{dBm}$

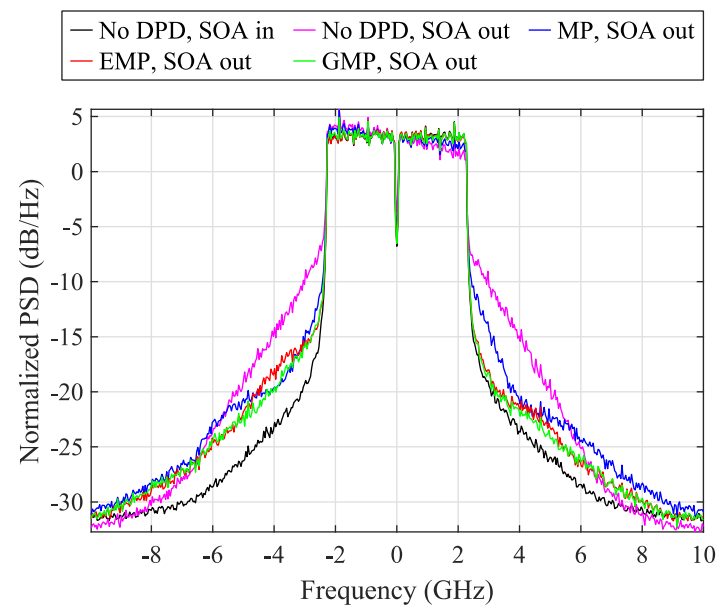

Fig. 5. Power spectral density at $P_{\text {ref }}=-14 \mathrm{dBm}$

Fig. 4 shows the 4-QAM constellations on the receiver side (in an optical back-to-back scenario) for an input power into the SOA $P_{r e f}=-14 \mathrm{dBm}$. Again qualitatively the ranking of the three DPD solutions in terms of performance is confirmed. Visually the effect is even more apparent than in Fig. 3.

Fig. 5 shows OFDM spectra for the same four scenarios. Note that frequency values are relative to the $1540 \mathrm{~nm}$ optical carrier. Also, since the plot is meant for comparison of SOA input and output, the magnitude scale is normalized hence the values are not physically representative. Observation of the in-band behavior shows that the DPD acts as a pre-equalizer (the tilt visible on the purple waveform is compensated). More interesting is the out-band behavior and one may see that adjacent channel power ratio (ACPR) is visibly reduced. In this respect GMP performs best (particularly visible on the left-hand side).

\section{B. DPD robustness}

A final comparative analysis is carried out and illustrated in Fig. 6 in terms of EVM (Fig. 6a) and NMSE (Fig. 6b). This time the parameters of the three predistorters are computed only once for a reference power of $-14 \mathrm{dBm}$. They are then used unaltered for system validation at various other input power levels in what is essentially a robustness analysis.
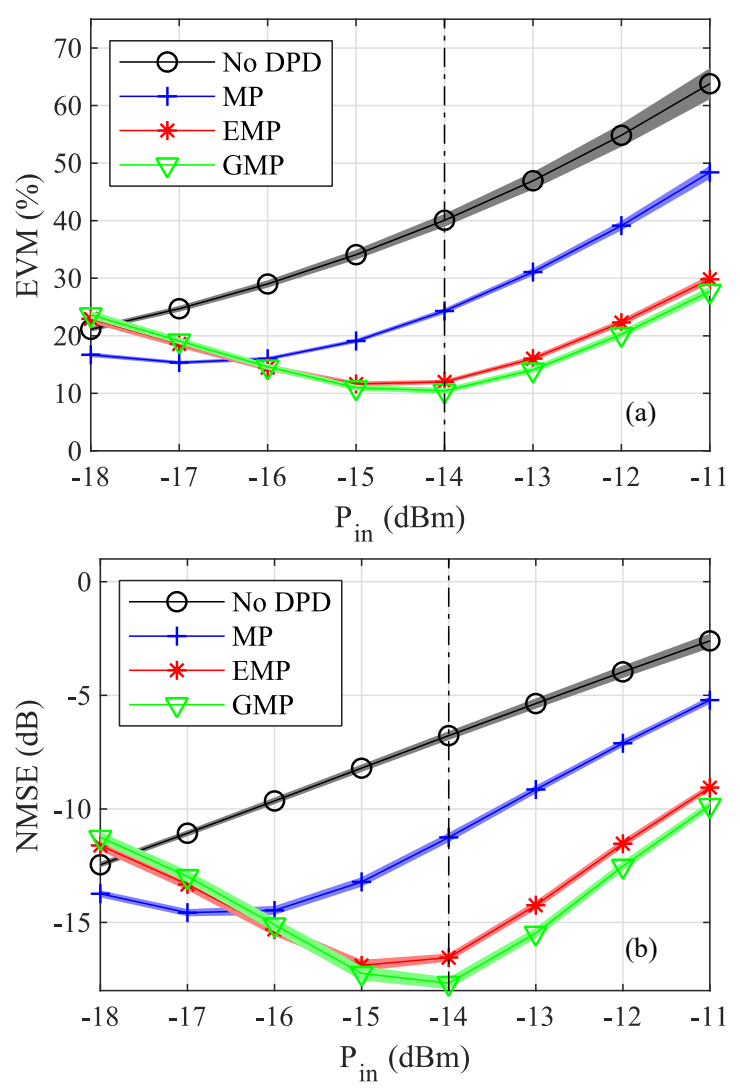

Fig. 6. Error Vector Magnitude (6.a) and Normalized Mean Square Error (6.b) at different SOA input powers, while using the three DPDs identified at $P_{\text {ref }}=-14 \mathrm{dBm}$

EMP and GMP clearly outperform MP, with GMP exhibiting a more robust behavior at higher power levels.

\section{CONCLUSION}

The present paper focuses on the use of generalized memory polynomials in the context of DPD for optical networks. Combined with a hill climbing algorithm used to select model parameters, the technique seems promising and the results in section IV show GMP outperforming other solutions. To the authors' knowledge this is the first time GMP predistortion is investigated for optical OFDM and the present conference paper is meant as a proof-of-concept. Future work is likely to include implementation of enhanced hill-climbing algorithms [9] and also focus on improving the simulation set-up by modeling the receiver more accurately and also taking into account a non-ideal channel.

\section{REFERENCES}

[1] F. M. Ghannouchi, O. Hammi, and M. Helaoui, Behavioral Modeling and Predistortion of Wideband Wireless Transmitters, 1st ed, John Wiley \& Sons, 2015.

[2] S. Amiralizadeh, A. T., Nguyen, and L. A. Rusch, "Modeling and compensation of transmitter nonlinearity in coherent optical OFDM," Optics express, vol. 23, no. 20, pp 26192-26207, September 2015.

[3] H. Schmuck et al., "Demonstration of an SOA-assisted open metroaccess infrastructure for heterogeneous services," Optics express, vol. 22, no. 1, pp. 737-748, January 2014.

[4] C. Chen, K. Batselier, M. Telescu, S. Azou, N. Tanguy, and N. Wong, "Tensor-network-based predistorter design for multiple-input multiple-output nonlinear systems," IEEE 12th International Conference on ASIC (ASICON), pp. 1117-1120, October 2017.

[5] C. Diouf, M. Younes, A. Noaja, S. Azou, M. Telescu, P. Morel, N. Tanguy, "Robustness analysis of a parallel two-box digital 
polynomial predistorder for an SOA-based CO-OFDM system," Optics Communications, vol. 402, pp. 442-452, November 2017.

[6] P. Jardin, and G. Baudoin, "Filter Lookup Table Method for Power Amplifier Linearization," IEEE Trans. on Vehicular Technology, vol. 56, no.3, pp. 1076-1087, May 2007.

[7] D. R. Morgan, Z. Ma, J. Kim, M. G. Zierdt, and J. Pastalan, "A generalized memory polynomial model for digital predistortion of RF power amplifiers," IEEE Trans. On Signal Processing, vol. 54, no. 10 , pp. 3852-3860, September 2006.

[8] S. Wang, M.A. Hussein, O. Venard, and G. Baudoin, "Optimal sizing of generalized memory polynomial model structure based on hillclimbing heuristic," IEEE 46th European Microwave Conference (EuMC), pp. 190-193, October 2016

[9] S. Wang, M.A. Hussein, O. Venard, and G. Baudoin, "A novel algorithm for determining the structure of digital predistortion models," IEEE Trans. on Vehicular Technology, vol. 67, no. 8, pp. 7326-7340, May 2018.

[10] H. Khaleghi, P. Morel, A. Sharaiha, and T. Rampone, "Experimental validation of numerical simulations and performance analysis of a coherent optical-OFDM transmission system employing semiconductor optical amplifier," J. Lightw. Technol., vol. 31, no. 1, pp. 161-170, January 2013.

[11] S. Bejan, S. Azou, P. Morel, M. Telescu, N. Tanguy, and A. Sharaiha, "A joint linearization/companding approach for improving a $\mathrm{CO}$ OFDM Transmitter,” IEEE Photon. Technol. Lett., vol. 27, no. 20, pp. 2162-2165, October 2015.

[12] R. Schmogrow et al., "Error vector magnitude as a performance measure for advanced modulation formats," IEEE Photonics Technol. Lett., vol. 24, no. 1, pp. 61-63, October 2011. 